\title{
Los costos de formación y la variación del número de partidos políticos en las provincias argentinas*
}

Party formation costs and the variation in the number

of political parties in Argentine provinces

\section{DANILO DEGIUSTTI}

Universidad de Buenos Aires, Argentina

ddegiustti@derecho.uba.ar

\section{GERARDO SCHERLIS}

Universidad de Buenos Aires, Argentina

Consejo Nacional de Investigaciones Científicas y Técnicas, Argentina

gscherlis@derecho.uba.ar

Argentina cuenta con un número comparativamente elevado de partidos políticos registrados. Esta situación es usualmente señalada como un problema para el sistema político, tanto en términos de representatividad como de gobernabilidad. Pese a ello, no se ha prestado hasta ahora atención a la heterogeneidad territorial de este fenómeno ni se ha intentado analizar sus causas en forma sistemática. Este artículo tiene por objetivo contribuir a cubrir este vacío. El artículo comienza por identificar una amplia heterogeneidad inter-distrital respecto a la cantidad de partidos reconocidos. Su principal hallazgo es que la diferencia en los requisitos que la ley exige para el reconocimiento de partidos políticos en las distintas provincias del país constituye la variable que mejor da cuenta de esta heterogeneidad. La investigación muestra a su vez que esta diferencia en la cantidad de partidos impacta en la cantidad de listas que se presentan para competir por cargos legislativos.

\section{Introducción}

Argentina cuenta con un número comparativamente elevado de partidos políticos registrados, al punto de contar con la mayor cantidad de partidos nacionales de América Latina. Esta situación es usualmente señalada como un problema para el sistema político, a partir del impacto que esto tendría en la fragmentación de la oferta electoral y en la de los bloques legislativos (Mustapic, 2013). A partir de ello, es habitual también que tanto los expertos

Articulo aceptado para su publicación el 10 de junio de 2019. 
(p. ej. Pomares et. al., 2012) como distintos sectores políticos (véase p. ej. Rosemberg, 2018) impulsen reformas electorales que se proponen como objetivo reducir el número de partidos.

Varios trabajos han abordado el tema de la fragmentación de la política argentina, asociándolo con el fenómeno de la desnacionalización partidaria (Leiras, 2010; Suárez Cao, 2011; Varetto, 2017; Gervasoni, 2018b). Como variables explicativas de estos fenómenos se han identificado, entre otras, la descentralización fiscal (Calvo y Escolar, 2005), la devaluación de las etiquetas partidarias y el consecuente desalineamiento electoral (Leiras, 2007) y un particularmente acentuado federalismo electoral (Abal Medina y Calvo, 2001; Mustapic, 2013).

Por su parte, algunos autores han identificado también la heterogeneidad territorial de la fragmentación partidaria. El trabajo de Navarro et al. (2013) pone el foco en esta heterogeneidad, a la que denomina fragmentación con desequilibrio regional, advirtiendo que la fragmentación partidaria «acontece de modo bien diverso entre jurisdicciones subnacionales (o provincias) en Argentina» (p. 41). Sin embargo, tal como concluye este mismo estudio, ninguna de las hipótesis presentes en la literatura respecto a la disparidad territorial de la fragmentación ofrece una explicación adecuada sobre este fenómeno (Navarro et al, 2013:61)

Por otra parte, todos los trabajos mencionados observan la fragmentación partidaria medida en términos del número efectivo de partidos. Aquí, en cambio, nos interesa poner el foco en el número de partidos registrados. De acuerdo al particular marco regulatorio de Argentina, a los fines de disputar elecciones por cargos legislativos nacionales, los partidos políticos deben obtener su reconocimiento en el distrito del país en el que pretenden competir. Esto hace que cada distrito pueda contar con un número diferente de partidos políticos y que, a su vez, cada distrito tenga su propia oferta electoral. Sin embargo, no contamos con trabajos que observen la disparidad en el número de partidos registrados en los diferentes distritos. Consecuentemente, tampoco ha habido intentos por comprender las causas de esta heterogeneidad específica.

Analizar la distribución territorial de los partidos registrados en las provincias argentinas es relevante porque, como surge de estudios anteriores (Clerici, 2016) y como se muestra también aquí, existe una alta correlación entre esta variable y la fragmentación de la oferta electoral. De modo que abordar las causas de la heterogeneidad territorial en el número de partidos registrados contribuye a comprender no solo la problemática de la cantidad de partidos sino también la de la así llamada sobre-oferta electoral, que afectarían a su vez el desempeño y la representatividad del sistema político argentino (Mustapic, 2013; Leiras, 2010). Identificar sus causas es asimismo 


\section{Danilo Degiustti y Gerardo Scherlis}

crucial a los efectos de estar en condiciones de proponer reformas a partir de un diagnóstico adecuado.

En este trabajo mostramos que el número de partidos reconocidos para competir por cargos legislativos nacionales en Argentina presenta una amplia variedad entre distritos. Nuestro principal hallazgo es que la variable más influyente en términos estadísticos sobre dicha variedad está constituida por los requisitos que la ley exige para el reconocimiento de partidos políticos en las distintas provincias del país. La investigación muestra a su vez que esta diferencia en la cantidad de partidos impacta en la cantidad de listas que se presentan para competir por cargos legislativos. En definitiva, la variación en las condiciones para registrar partidos es el factor que muestra un mayor grado de influencia sobre la disparidad en la cantidad de partidos entre distritos mientras que, tal como cabría esperar, la cantidad de partidos registrados impacta a su vez sobre la variación en la oferta electoral.

El trabajo se organiza del siguiente modo. Tras esta introducción, la segunda parte describe los requisitos cuantitativos para registrar partidos políticos en Argentina y muestra la heterogeneidad en la cantidad de partidos registrados en los 24 distritos del país. La tercera sección discute las posibles causas de la disparidad inter-distrital y destaca el efecto de los requisitos cuantitativos para formar partidos, es decir el efecto de los costos de formación. La cuarta sección ofrece evidencia respecto a que el número de partidos reconocidos impacta a su vez sobre la composición de la oferta electoral. La quinta sección concluye sintetizando los principales hallazgos del trabajo y sus implicancias.

\section{Los requisitos para crear partidos en Argentina y la heterogeneidad interdistrital}

Los requisitos para crear y conservar partidos en Argentina están regulados por la Ley Orgánica de los Partidos Políticos No 23.298. Una primera distinción prevista en la norma es aquella que diferencia a los partidos de distrito de los partidos de orden nacional. El partido de distrito es aquel que obtiene su personería jurídico-política precisamente en alguno de los 24 distritos electorales del país, es decir en alguna de las 23 provincias o en la Ciudad Autónoma de Buenos Aires (CABA).

Esta personería habilita al partido de distrito para presentar listas de candidatos a senadores y diputados nacionales. El partido de distrito (o la reunión de varios partidos de distrito en una alianza) es la única forma jurídica mediante la cual se pueden presentar listas de candidatos en las 
mencionadas categorías de cargos, categorías para los cuales el distrito electoral justamente coincide con cada una de las provincias y CABA.

Los partidos nacionales, por su parte, son aquellos que han logrado ser reconocidos como partido de distrito bajo una misma denominación en cinco o más distritos del país. El reconocimiento como partido nacional habilita a postular candidatos para presidente y vicepresidente de la Nación, categoría que se disputa en un distrito único conformado por todo el territorio de la Nación. El reconocimiento como partido nacional no habilita a presentar candidaturas a legisladores nacionales en todos los distritos, facultad que solo tienen los partidos de distrito que lo integran allí donde están registrados.

De acuerdo a la legislación nacional, entonces, las organizaciones partidarias surgen necesariamente como partidos de distrito, y es la reunión de cinco o más partidos de distrito bajo una misma etiqueta lo que lleva a la formación de un partido nacional.

Como se observa en la Tabla 1, no debe confundirse esta distinción entre partidos de distrito y partidos nacionales con la que distingue a estos dos de la categoría que puede denominarse «de orden provincial». Esta última no está contemplada en la normativa nacional, sino en cada uno de los regímenes jurídicos de las provincias y de la Ciudad de Buenos Aires, y constituye la forma jurídica a través de la cual los partidos compiten por cargos de nivel sub-nacional: gobernaciones y legislaturas provinciales, intendencias y concejos deliberantes, además de otros cargos que pueden estar previstos en las legislaciones provinciales. A menudo el partido de distrito y el partido provincial son una misma organización, con iguales miembros y dirigentes, pero conforman dos personas jurídicas diferenciadas. El partido de distrito obtiene su reconocimiento ante el juez federal con competencia electoral del distrito, mientras que en el orden provincial los partidos son reconocidos por las autoridades electorales de la provincia (tribunal electoral provincial, junta electoral de la provincia, o las que las instituciones provinciales dispongan) (Dalla Via, 2010:176-7) ${ }^{1}$.

Las legislaciones provinciales en general también contemplan un cuarto nivel de partidos, el municipal, que habilita a competir por cargos en el municipio en el que se obtiene el reconocimiento (típicamente para el cargo de intendente y concejal) compartiendo dicha facultad con los partidos provinciales, que pueden competir en todos los municipios de la provincia. 


\section{Tabla 1}

Argentina: Los distintos niveles de partidos políticos

\begin{tabular}{|l|l|l|l|}
\hline Tipo de partido & Cargos a los que postula & $\begin{array}{l}\text { Legislación que lo } \\
\text { regula }\end{array}$ & $\begin{array}{l}\text { Autoridad } \\
\text { competente para el } \\
\text { reconocimiento }\end{array}$ \\
\hline Partido de Distrito & $\begin{array}{l}\text { Diputados y Senadores } \\
\text { nacionales }\end{array}$ & Nacional. Ley 23.298 & $\begin{array}{l}\text { Juez federal del } \\
\text { distrito }\end{array}$ \\
\hline Partido Nacional & $\begin{array}{l}\text { Presidente y Vicepresidente } \\
\text { de la Nación }\end{array}$ & Nacional. Ley 23.298 & $\begin{array}{l}\text { Juez federal del } \\
\text { distrito de su } \\
\text { fundación }\end{array}$ \\
\hline Partido provincial & $\begin{array}{l}\text { Gobernador, legisladores y } \\
\text { demás cargos provinciales y } \\
\text { municipales previstos en cada } \\
\text { provincia y CABA }\end{array}$ & $\begin{array}{l}\text { Provincial. Cada } \\
\text { provincia tiene su } \\
\text { legislación específica }\end{array}$ & $\begin{array}{l}\text { Autoridades } \\
\text { electorales } \\
\text { provinciales }\end{array}$ \\
\hline
\end{tabular}

Fuente: Elaboración propia en base a la Ley 23.298 y legislación provincial.

La distinción entre partido de distrito, partido nacional y partido provincial es una particularidad excepcional de la regulación argentina en el contexto latinoamericano (Scherlis, 2016). Algunos países, como Brasil y Uruguay, solo admiten partidos de orden nacional, habilitados para competir por todos los cargos en disputa en todo el territorio del país. Pero la mayoría de los países de la región distinguen entre partidos nacionales y subnacionales (a los que se denomina provinciales, estaduales o regionales, según el país). Los nacionales están siempre habilitados para postular candidatos a cargos nacionales - ejecutivos y legislativos - en todo el territorio del país, y en algunos casos (por ejemplo México) también para cargos sub-nacionales. Los partidos sub-nacionales compiten por cargos de naturaleza sub-nacional (estadual, provincial, departamental o regional, según el país). Entre las democracias de la región solo Argentina y Costa Rica contemplan la posibilidad de que partidos reconocidos en un solo distrito puedan postular listas de candidatos para cargos legislativos nacionales. Pero solo en Argentina la legislación confiere en forma exclusiva la competencia para la postulación de candidatos a cargos legislativos nacionales a partidos con reconocimiento en el distrito (Scherlis, 2016).

Los partidos de distrito pueden a su vez formar parte de un partido nacional, tanto como pueden no hacerlo. En el total de partidos de distrito 
registrados en enero de 2018, 274 no eran parte de un partido nacional y 386 sí. Es decir que si bien una parte mayoritaria de los partidos de distrito es parte de un partido nacional, son muchos los partidos de distrito que se constituyen específicamente para competir por los cargos en una provincia determinada. En cambio, como surge de lo hasta aquí dicho, no puede haber un partido nacional que no esté conformado por partidos de distrito.

Todo lo anterior explica por qué al momento de analizar los requisitos para crear partidos en Argentina el foco se dirige normalmente hacia los partidos de distrito (Mustapic, 2013; Su, 2015). Veamos entonces ahora cuáles son estos requisitos.

Para constituir y conservar un partido de distrito se requiere contar con un número de afiliados no inferior al 0,4 por ciento del total del padrón de electores de la provincia, hasta el máximo de un millón de electores. Esto significa que el número máximo de afiliados requerido, para aquellos distritos que superen el millón de votantes, es de 4.000.

A su vez, como es habitual en la legislación electoral comparada, existe también un requisito de representatividad electoral para mantener el registro como partido de distrito, que en este caso consiste en alcanzar el 2\% de los votos en al menos una de cada dos elecciones en la provincia respectiva. $\mathrm{Al}$ no darse mayores precisiones al respecto, se entiende que los partidos alcanzan este porcentaje también cuando forman parte de una alianza que obtiene esa cantidad de votos en el distrito.

Los estudios comparados muestran que estos requisitos suponen un nivel relativamente bajo de exigencia (Zovatto, 2006; Scherlis, 2014) y advierten que esta flexibilidad para registrar y conservar partidos contribuye a la existencia de un elevado número de organizaciones partidarias (Straface y Mustapic, 2009; Abal Medina, 2010; Mustapic et. al., 2011). Este análisis suele ser compartido por los observadores y los propios políticos². Si bien cada año se producen variaciones en el número total de partidos de distrito reconocidos en el país, desde el año 2002 el total de éstos se ha mantenido en general por encima de los $600^{3}$. En enero de 2018 la Cámara Nacional Electoral reportaba la existencia de 660 partidos de distrito reconocidos. Este número supone un promedio de 26,8 partidos por cada uno de los 24 distritos del país.

No obstante, lo que interesa remarcar en este artículo es que la cantidad de partidos de distrito se distribuye de forma muy desigual a lo largo de estos 24 distritos, como se puede apreciar en el Gráfico 1.

2 Véase por ejemplo: «Laura Alonso: no puede ser que de 600 partidos 450 sean sellos de goma», Diario Perfil, 7 de agosto de 2018.

3 La excepción se da en los años 2011, 2012 y 2013, cuando por aplicación de una reforma que obligó a los partidos a ser más estrictos en cuanto a la reunión de la cantidad de afiliados, el número de partidos se ubicó entre los 500 y 550 . Sin embargo pronto volvió a crecer hasta superar la barrera de los 600 . 


\section{Danilo Degiustti y Gerardo Scherlis}

\section{Gráfico 1}

Partidos de distrito reconocidos, por provincia

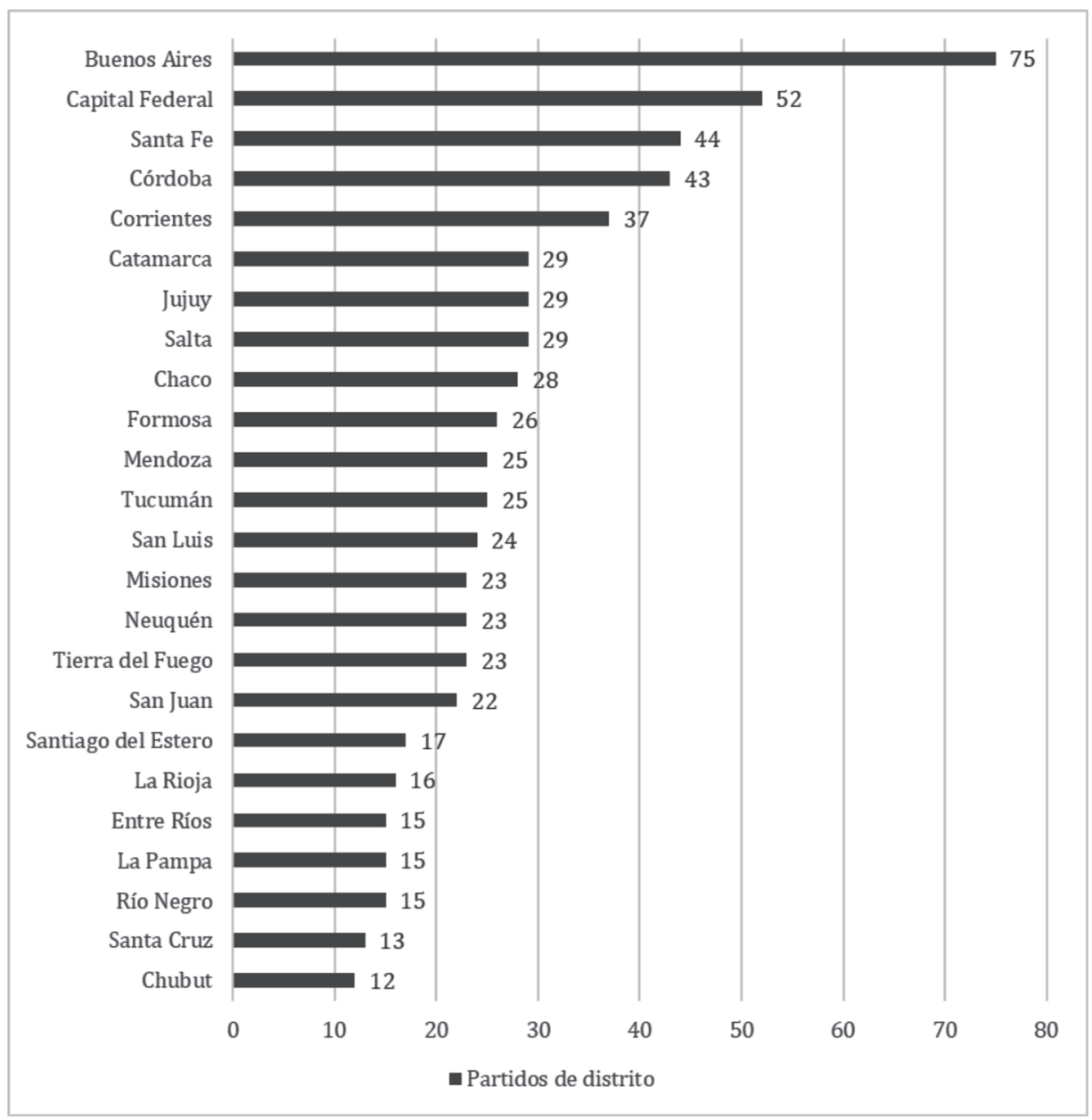

Fuente: elaboración propia en base a datos de la Cámara Nacional Electoral al 15/01/2018.

Como ya se señaló, entre las variadas implicancias que se siguen del particular marco regulatorio de los partidos políticos argentinos, se destaca el hecho de que cada provincia tiene un número diverso de partidos reconocidos para competir por los cargos legislativos nacionales.

La provincia de Buenos Aires encabeza la lista con 75 partidos de distrito inscriptos, seguida por la Ciudad de Buenos Aires, con 52. En el otro extremo, 
la provincia de Chubut cuenta con 12 partidos de distrito y la de Santa Cruz con 13 .

Esta sencilla observación pone en evidencia que el promedio de casi 27 partidos por distrito oculta una realidad que merece ser contemplada y analizada. Afirmar que en el país hay más de 600 partidos, o que en promedio cada distrito tiene 27, no permite aprehender la marcada heterogeneidad que supone la existencia de algunos distritos con 12, 13 o 15 partidos y de otros con más de 50.

Sin embargo, hasta donde sabemos, no existen trabajos que hayan observado sistemáticamente estas diferencias y consecuentemente tampoco se ha indagado sobre sus causas. La pregunta que sigue es, precisamente, qué factores explican la heterogeneidad en la cantidad de partidos reconocidos en los diferentes distritos de la República Argentina.

\section{Las causas de la variedad interprovincial}

En tiempos recientes diversos estudios han introducido las barreras legales para la competencia, o los así llamados costos de formación de partidos $(\mathrm{CFP})^{4}$ como una variable que podría contribuir a explicar el número de partidos en los sistemas políticos (p. ej. Van Biezen y Rashkova, 2014; Tavits, 2006; Birnir, 2004). Para el caso de los países latinoamericanos, $\mathrm{Su}$ (2015) precisamente ha mostrado que los requisitos legales para competir constituyen una variable significativa a la hora de explicar el número absoluto de partidos que compiten en elecciones y también el número efectivo de partidos.

En este mismo sentido, consideramos que los CFP podrían operar como una variable explicativa de la variación del número de partidos registrados en las provincias argentinas. Nuestra hipótesis sugiere precisamente que, a nivel distrito, cuanto mayor sea el porcentaje de afiliados requerido para conformar partidos, menor será el número de partidos reconocidos.

Es notable que, en lo formal, estos requisitos son idénticos para todos los distritos. De hecho, en todos los casos surgen de un mismo texto jurídico, el Artículo 7 bis de la Ley Orgánica de los Partidos Políticos $\mathrm{N}^{\circ} 23.298$, el cual establece que los partidos deben acreditar la «afiliación de un número de electores no inferior al cuatro por mil (4\%) del total de los inscriptos en el registro de electores del distrito correspondiente, hasta el máximo de

4 El concepto de costo de formación de los partidos es tomado del trabajo de Simon Hug (2001). 
un millón (1.000.000)», condición que deben mantener para conservar la personería (art. $7^{\circ}$ ter).

Sin embargo, esto significa implícitamente que en aquellos distritos que superen el millón de electores, el requisito del 4 por mil (o de 0,4 por ciento) sobre el padrón de electores deviene en un requisito de 4.000 afiliaciones, independientemente de qué porcentaje de afiliaciones suponga ese número sobre el padrón del distrito. Esta distinción se aplica en los hechos a los siete distritos más poblados del país: Buenos Aires, Ciudad de Buenos Aires, Córdoba, Entre Ríos, Mendoza, Santa Fe y Tucumán.

En estos casos el porcentaje efectivo de electores que se necesita es menor al 0,4 por ciento que rige para los demás distritos. La Provincia de Buenos Aires presenta el caso extremo. Dado que multiplica en más de doce veces el millón de electores, el requisito general del 0,4 por ciento deviene en una exigencia de contar con el 0.03 por ciento de afiliados. En otras palabras, si se cumpliera con el 0,4 por ciento requerido como fórmula general en la Ley, en la Provincia de Buenos Aires sería necesario reunir 49.005 afiliados en lugar de los 4.000 efectivamente requeridos.

En medida decreciente, distorsiones similares se presentan en el resto de los distritos que superan el millón de afiliados. La Tabla 2 muestra el número absoluto y el porcentaje efectivo de afiliados que debe reunirse en cada una de las provincias argentinas para constituir un partido de distrito.

En las siete provincias de mayor población del país el porcentaje de afiliaciones requerido es variable, desde el 0,03 por ciento de la Provincia de Buenos y en segundo lugar el 0,14 de Córdoba hasta el 0,37 de la provincia de Entre Ríos. Solo a partir de la octava provincia argentina de mayor población, Salta, resulta efectivo y constante el porcentaje de 0,4 por ciento ${ }^{5}$. Cuanto mayor es el electorado de un distrito, más accesible en términos relativos resulta cumplir con el requisito de afiliaciones.

Una observación superficial permite fácilmente asociar la diferencia en el porcentaje de afiliaciones requeridas con el número de partidos reconocidos. Si se toman las 17 provincias en las que el porcentaje del $0,4 \%$ es efectivo, el promedio de partidos reconocidos es de 22,4 . Si en cambio se toman las cuatro provincias más pobladas, donde los porcentajes efectivos son sustancialmente menores, el promedio de partidos por distrito es de 54 .

Un mínimo aumento del electorado salteño supondrá para próximos años que esta provincia también disminuya su porcentaje relativo de afiliaciones requeridas. 
Revista SAAP $\cdot$ Vol. 13, № 1

Tabla 2

Porcentaje de electores y afiliados requeridos, por provincia.

\begin{tabular}{|c|c|c|c|}
\hline Provincia & Electores 2017 & $4 \%$ (hasta $1 \mathrm{M}$ de electores) & $\%$ efectivo \\
\hline Buenos Aires & 12.251 .329 & 4000 & 0,033 \\
\hline Córdoba & 2.884 .427 & 4000 & 0,139 \\
\hline Santa Fe & 2.715 .615 & 4000 & 0,147 \\
\hline CABA & 2.555 .490 & 4000 & 0,157 \\
\hline Mendoza & 1.407 .780 & 4000 & 0,284 \\
\hline Tucumán & 1.217 .274 & 4000 & 0,329 \\
\hline Entre Ríos & 1.077 .127 & 4000 & 0,371 \\
\hline Catamarca & 311.027 & 1242 & 0,4 \\
\hline Corrientes & 845.466 & 3382 & 0,4 \\
\hline Chaco & 912.052 & 3631 & 0,4 \\
\hline Chubut & 435.144 & 1728 & 0,4 \\
\hline Formosa & 442.941 & 1780 & 0,4 \\
\hline Jujuy & 537.729 & 2144 & 0,4 \\
\hline La Pampa & 282.012 & 1128 & 0,4 \\
\hline La Rioja & 281.459 & 1122 & 0,4 \\
\hline Misiones & 894.679 & 3588 & 0,4 \\
\hline Neuquén & 488.309 & 1950 & 0,4 \\
\hline Río Negro & 536.983 & 2145 & 0,4 \\
\hline Salta & 991.299 & 3968 & 0,4 \\
\hline San Juan & 554.299 & 2215 & 0,4 \\
\hline San Luis & 376.898 & 1510 & 0,4 \\
\hline Santa Cruz & 248.334 & 973 & 0,4 \\
\hline Santiago & 735.480 & 2935 & 0,4 \\
\hline Tierra del Fuego & 132.924 & 531 & 0,4 \\
\hline
\end{tabular}

Fuente: elaboración propia en base a datos de la Cámara Nacional Electoral. 


\section{Diseño metodológico}

A los fines de testear nuestra hipótesis, utilizamos una base de datos que comprende a los 24 distritos del país (las 23 provincias y la Ciudad Autónoma de Buenos Aires). Cada uno de ellos es una unidad de análisis, por lo cual contamos con un número de casos relativamente pequeño. La variable dependiente es el número de partidos de distrito reconocidos por la Justicia Nacional Electoral en cada distrito al 15 de enero de 2018. La variable independiente de interés es el porcentaje de afiliados requeridos para conservar la personería política en relación con el total de electores en cada distrito, que presenta una variación entre 0,03 y 0,4 .

Dado que el número de partidos puede ser consecuencia de muchos otros factores, es necesario controlar por otras variables, para así estimar mejor el efecto de la variable de interés.

Existe una extensa literatura sobre los factores que impactan en el número de partidos. En términos generales, estas variables pueden dividirse en las de carácter sociológico y las institucionales. El primer grupo enfatiza la preexistencia de conflictos, fisuras o clivajes presentes en la sociedad, asumiendo que una mayor fragmentación social (típicamente en términos de clase, religión o etnia) debería dar lugar a un mayor número de partidos que expresen esa diversidad. El segundo pone el foco en cómo el diseño institucional moldea los incentivos coalicionales para participar de la vida electoral. Aquellos esquemas institucionales que exigen una mayor agregación de voluntades para ganar elecciones u obtener acceso a los cargos deberían asociarse a un menor número de partidos (Amorim Neto y Cox, 1995; Taagepera, 1999).

Nuestra variable de interés se vincula, obviamente, con el grupo de variables institucionales: cuanto más alto sea el porcentaje de afiliaciones necesarias para la inscripción, mayor es el costo de entrada al juego electoral $(\mathrm{Su}, 2015)$.

Pero también otros factores presentes en la literatura podrían impactar sobre el número de partidos. En el marco de la comparación entre distritos de la República Argentina consideramos necesario incorporar las siguientes variables de control:

Magnitud de distrito: La cantidad de cargos que se eligen por distrito es una variable institucional usualmente considerada decisiva para explicar el número de partidos (Ordeshook y Shvetsova, 1994). Su importancia radica en que determina el porcentaje de votos que un partido necesita para obtener representación en el parlamento, especialmente cuando, como en este caso, la fórmula electoral de distribución de los cargos se mantiene constante entre las unidades de análisis. Particularmente para el caso argentino se ha 
argumentado que la baja magnitud de los distritos posee un efecto reductor sobre la fragmentación (Calvo et al, 2001). En definitiva, la expectativa con respecto a esta variable es que mientras más cargos haya para repartir en un distrito, mayores serán los incentivos para crear partidos políticos.

Padrón electoral: Debido a que el requisito formal del porcentaje de afiliaciones es en gran medida una derivación de la cantidad de votantes registrados en cada distrito, capturar más precisamente su efecto requiere introducir como variable de control el padrón electoral de cada provincia para el año 2017. Si bien las diferencias en el padrón pueden en parte ser capturadas por la variable magnitud de distrito, esta última no resulta del todo precisa por la fuerte sobre- y subrepresentación distrital existente en Argentina. ${ }^{6}$

Simultaneidad de elecciones: Diversos estudios sobre América Latina han demostrado que la concurrencia de elecciones presidenciales y legislativas lleva a menor fragmentación del sistema partidario $(\mathrm{Su}, 2015$; Shugart and Carey, 1992, Jones 1995). En el caso argentino, también se ha observado que el número de partidos que compiten para elegir diputados nacionales se reduce cuando la elección legislativa nacional coincide con la elección para elegir gobernador provincial (Jones, 1997). De modo que introducimos como variable de control la observación respecto a la simultaneidad entre elecciones. Dado que el último ciclo de elecciones para gobernador tuvo lugar en el año 2015, observamos si la elección a diputados nacionales realizada ese año coincidió en cada distrito con la elección para gobernador provincial. En el caso de dos provincias, Corrientes y Santiago del Estero, que tuvieron la elección de gobernador en 2017, observamos la presencia de simultaneidad o no en las elecciones de diputados de ese año. La expectativa es que en aquellos distritos donde las elecciones para los cargos legislativos nacionales coinciden con la elección del ejecutivo provincial se produzca una reducción en el número de partidos inscriptos en relación con los distritos donde ambas elecciones se realizan en forma separada.

Nivel de democracia subnacional: En los últimos años se ha demostrado la existencia de variaciones significativas en los niveles de democracia entre las distintas unidades subnacionales de Argentina (Gervasoni, 2018a; Giraudy, 2010). Es razonable plantear como expectativa teórica que en aquellas provincias con menor nivel de democracia subnacional existan menores incentivos para la creación de nuevos partidos políticos. El bajo nivel de democracia supone un bajo nivel de competitividad electoral, ya que dichas provincias tienden a estar dominadas por un partido político que controla los resortes del poder político y, por diversos medios, reduce las posibilidades y en consecuencia también los incentivos para que surja una oposición competitiva. Podemos esperar entonces que cuanto menor sea el nivel de

$6 \quad$ Agradecemos al evaluador la sugerencia de incluir esta variable. 


\section{Danilo Degiustti y Gerardo Scherlis}

democracia subnacional sea asimismo menor el número de partidos políticos. Por otro lado, y en sentido opuesto, la experiencia política argentina también ofrece ejemplos de partidos dominantes en distritos de baja competitividad electoral que se fragmentan a los fines de presentarse ante el electorado como opciones alternativas y capturar así una mayor cantidad de cargos que los que obtendrían en caso de presentarse como una única fuerza política. En relación con ello, las condiciones de predominio ofrecen asimismo escenarios propicios para que las fuerzas políticas gobernantes en las provincias desplieguen estrategias de segmentación estratégica (Escolar, 2014), propiciando por ejemplo la presentación de una oferta electoral fragmentada en cierto nivel de competencia pero acopladas a través de las así llamadas «listas colectoras» en otra categoría. Un uso extendido de este tipo de estrategias implicaría una proliferación mayor de partidos registrados en distritos con bajo nivel de democracia subnacional. En cualquier caso, creemos conveniente la inclusión de esta variable como control, para lo cual utilizamos el índice de democracia subnacional (Gervasoni, 2009).

Antigüedad de la provincia: Los 24 distritos que consideramos en el análisis comenzaron a elegir autónomamente a sus autoridades en diferentes momentos históricos. Algunos distritos -como por ejemplo Córdoba, La Rioja, Tucumán, Santa Fe, o la Provincia de Buenos Aires- lo hicieron desde la propia constitucionalización del país, en el siglo XIX. Otros, como Chaco, Misiones, La Pampa, Chubut o Neuquén, recién fueron reconocidos como provincias, y por lo tanto como distritos autónomos, en los años $50 \mathrm{~s}$ del siglo XX. Tierra del Fuego solo comenzó a elegir sus propias autoridades en 1991 y, finalmente, la Ciudad de Buenos Aires lo hizo en 1996. El tiempo transcurrido desde que un sistema político comienza a funcionar para elegir autoridades mediante elecciones podría influir también en el número de partidos actuantes. La afirmación y protección de la identidad regional o provincial puede ser un valor político central para segmentos del electorado, que desearán confiar su representación a partidos que se organizan con este propósito (Leiras, 2010:219). En esta línea, un trabajo sobre cuatro países latinoamericanos describe cómo a partir de la elección directa de gobernadores y alcaldes, los partidos de procedencia local y regional han crecido en número e influencia debilitando a los partidos nacionales (Sabatini, 2003). La expectativa teórica en este marco es que cuanto mayor sea la longevidad de un distrito, más fuerte será la identidad política provincial y, por ende, cabe esperar que también sean mayores los incentivos para la creación y permanencia de partidos de base sub-nacional.

Índice de Desarrollo Humano: La literatura sugiere que la fragmentación partidaria en Argentina es mayor en las provincias centrales y menor en las periféricas (Calvo y Escolar, 2005; Malamud y De Luca, 2016). Esta distinción 
se vincula por un lado con la magnitud del distrito (en general mayor en las provincias centrales y menor en las periféricas), pero también con el nivel de desarrollo. En los términos de Navarro et al «la FDR [fragmentación partidaria con desequilibrio regional] plantea un escenario de fragmentación partidaria que afecta especialmente a las regiones más desarrolladas de Argentina» (2013:44). Consideramos por ello pertinente introducir como variable de control el Índice de Desarrollo Humano por provincia (2016), que captura indicadores de salud, educación y riqueza.

La incorporación de esta variable de control se justifica asimismo desde una perspectiva pluralista de la democracia (Dahl, 1991), según la cual cabe esperar que los diversos intereses que surgen en las complejas sociedades modernas se agrupen libremente a los fines de influir sobre la toma de decisiones políticas. Esto puede darse a través de la conformación de grupos de interés y asociaciones de diversa índole, pero también conformando partidos políticos. Desde esta perspectiva, cabría esperar que en el marco de sistemas electorales similares, en sociedades más modernas o desarrolladas, con mayor complejidad de intereses, surjan más partidos que en sociedades con características más tradicionales.

Finalmente, debemos considerar que los partidos de distrito tienen como base precisamente una provincia, y que pueden estar por lo tanto influidos por la dinámica propia del sistema político provincial respectivo. Como ya se mencionó, cada uno de los 24 distritos del país tiene una dinámica específica en lo que hace a la competencia por el poder político provincial, y esta dinámica por cierto podría influenciar en el número de partidos que se registran para competir por los cargos legislativos nacionales en el distrito. Para controlar la influencia del sistema de partidos provincial, tomamos como variable el número de agrupaciones que se presentaron a las elecciones de diputados provinciales en 2015 en cada distrito, con la expectativa de que un mayor número de agrupaciones en competencia en el orden provincial se asocie con un mayor número de partidos inscriptos en el orden federal.

Otras variables de control que han sido utilizadas por la literatura como la existencia de subsidios públicos a los partidos, el voto preferencial, el crecimiento del PBI, la inflación y la edad de la democracia (por ejemplo Su, 2015), en este caso fueron descartadas al mantenerse constantes para todos los distritos.

Nuestra variable dependiente es de conteo, el número total de partidos reconocidos en el distrito, ya que no incluye números negativos ni decimales. Para este tipo de variable se utilizan modelos de regresión Poisson y binomial negativo. La media de la variable dependiente es 27.5 , mientras que la varianza (el cuadrado de la desviación estándar) es de 206,2. Dado que la desviación estándar es varias veces superior a la media, para nuestro modelo una distribución binomial negativa es más adecuada que una Poisson (que 


\section{Danilo Degiustti y Gerardo Scherlis}

supone una media igual a la varianza). De todos modos, presentamos resultados para ambos modelos.

\section{Resultados}

La Tabla 3 reporta los resultados de la regresión binomial negativa (modelo1) y de la regresión Poisson (modelo 2). Los resultados de ambos modelos brindan apoyo a nuestra hipótesis, mostrando que el requisito de afiliaciones tiene un efecto negativo y significativo sobre el número de partidos reconocidos, por lo cual a medida que aumenta el porcentaje de afiliaciones sobre electores requerido en un distrito, esperamos que disminuya la cantidad de partidos reconocidos.

\section{Tabla 3}

Efectos sobre el número de partidos reconocidos por distrito

\begin{tabular}{|c|c|c|}
\hline Variable & $\begin{array}{c}\text { Modelo 1 } \\
\text { (Binomial negativo) }\end{array}$ & $\begin{array}{l}\text { Modelo } 2 \\
\text { (Poisson) }\end{array}$ \\
\hline Afiliaciones & $\begin{array}{c}-2.267 * * \\
(0.879)\end{array}$ & $\begin{array}{c}-2.273 * * \\
(0.870)\end{array}$ \\
\hline IDH & $\begin{array}{l}-6.607 * * \\
(2.506)\end{array}$ & $\begin{array}{l}-6.540 * \\
(2.600)\end{array}$ \\
\hline Democracia subnacional & $\begin{array}{l}-0.002 \\
(0.011)\end{array}$ & $\begin{array}{l}-0.002 \\
(0.011)\end{array}$ \\
\hline Antigüedad de la provincia & $\begin{array}{c}0.000 \\
(0.000)\end{array}$ & $\begin{array}{c}0.000 \\
(0.000)\end{array}$ \\
\hline Magnitud de distrito & $\begin{array}{l}0.120 * \\
(0.056)\end{array}$ & $\begin{array}{l}0.119 * \\
(0.056)\end{array}$ \\
\hline Padrón & $\begin{array}{l}-2.75^{*} \\
(1.40)\end{array}$ & $\begin{array}{l}-2.73 \\
(1.41)\end{array}$ \\
\hline $\begin{array}{l}\text { Simultaneidad de elección } \\
\text { a gobernador }\end{array}$ & $\begin{array}{l}-0.190 \\
(0.136)\end{array}$ & $\begin{array}{l}-0.189 \\
(0.137)\end{array}$ \\
\hline $\begin{array}{l}\mathrm{N} \text { de agrupaciones para } \\
\text { diputados provinciales }\end{array}$ & $\begin{array}{c}0.002 \\
(0.005)\end{array}$ & $\begin{array}{c}0.002 \\
(0.004)\end{array}$ \\
\hline Constante & $\begin{array}{c}9.397 * * * \\
(2.076)\end{array}$ & $\begin{array}{c}9.346^{* * *} \\
(2.174)\end{array}$ \\
\hline Log likelihood & -75.03 & -75.05 \\
\hline Alpha & $\begin{array}{c}0.003 \\
(0.021)\end{array}$ & \\
\hline Wald chi2 (8) & 33.39 & 920.75 \\
\hline Prob > chi2 & 0.000 & 0.000 \\
\hline Observaciones & 24 & 24 \\
\hline Pseudo R2 & 0.194 & 0.442 \\
\hline
\end{tabular}

Errores estándar robustos entre paréntesis. ${ }^{*} \mathrm{p}<0.05 * * \mathrm{p}<0.01 * * * \mathrm{p}<0.001$. 
En cuanto a las variables de control, la magnitud de distrito muestra un efecto positivo y estadísticamente significativo con un nivel de confianza del 95\% para los dos modelos, en línea con la literatura (Ordeshook y Shvetsova, 1994; Calvo et. al. 2001) y con nuestras propias expectativas. Es decir que un aumento en la magnitud de distrito produce un aumento del número esperado de partidos reconocidos. Contra las expectativas teóricas, la variable IDH muestra un efecto negativo y estadísticamente significativo, con un nivel de confianza de $99 \%$ en el primer modelo y del $95 \%$ en el segundo. También a la inversa de lo esperado, el padrón electoral muestra un efecto negativo en ambos modelos, pero sólo resulta significativo al 95\% para el binomial negativo. El resto de las variables de control no muestran efectos estadísticamente significativos.

Al observar nuestra variable independiente de interés, el porcentaje requerido de afiliaciones, encontramos que esta es la que muestra mayor significancia estadística en ambos modelos, con un nivel de confianza del $99 \% \%^{7}$. Para entender mejor este efecto sustantivo presentamos el Gráfico 2 que, utilizando una técnica de simulación, predice el número de partidos reconocidos a diferentes niveles de porcentaje de firmas, manteniendo el resto de las variables en su media.

Del gráfico se desprende un claro declive en el número predicho de partidos reconocidos a medida que aumenta el número de firmas/afiliaciones requerido. Por ejemplo, si una provincia exige el 0,1 por ciento de afiliaciones, manteniendo en su media las variables de control, esperamos que tenga en promedio 44 partidos reconocidos; mientras que si en otra se exige 0,4 por ciento de afiliaciones, esperamos que en promedio el número de partidos sea apenas superior a 20 .

El caso de la provincia de Buenos Aires explica en gran parte la fortaleza de la regresión ya que presenta la correlación más extrema entre menor porcentaje requerido y mayor número de partidos. Sin embargo, esta correlación se verifica también ostensiblemente en Ciudad de Buenos Aires, Córdoba y Santa Fe, los tres distritos que siguen a la provincia de Buenos Aires tanto en términos de requisitos como de cantidad de partidos. 


\section{Gráfico 2.}

Predicción del número de partidos reconocidos

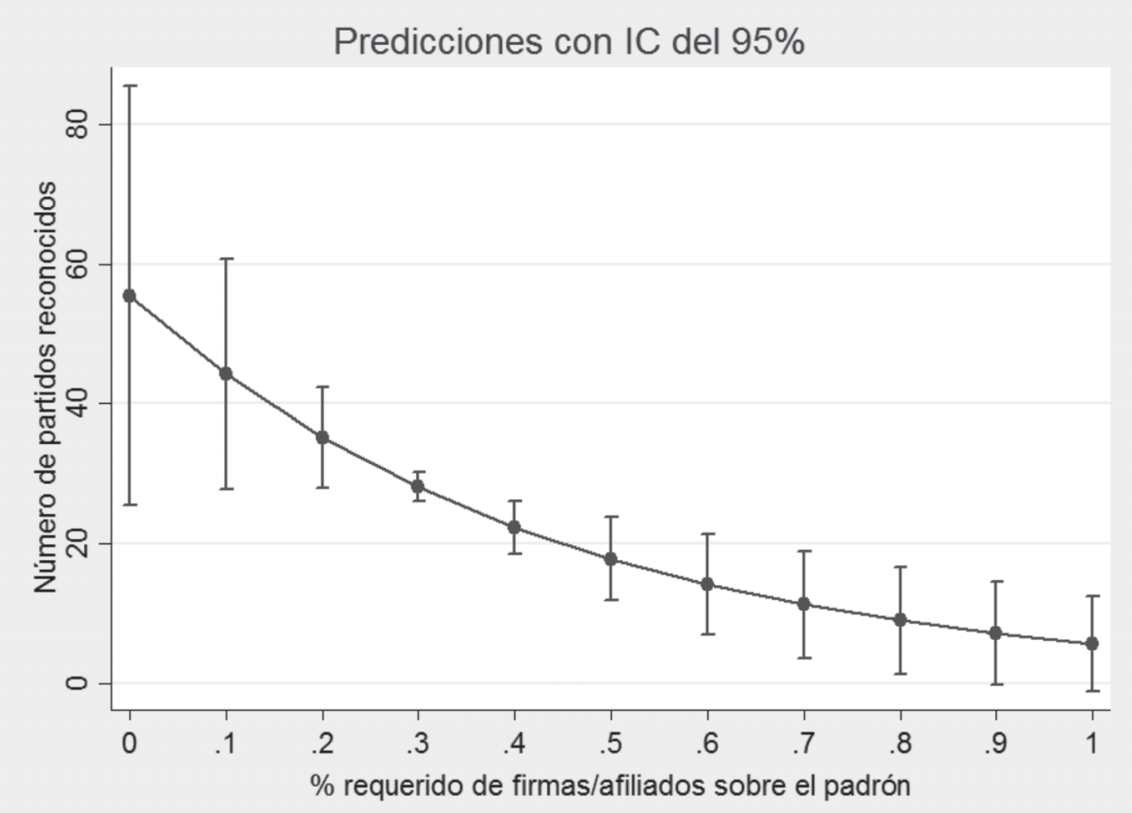

Fuente: elaboración propia.

Una de las objeciones que suelen hacerse a las explicaciones basadas en las reglas electorales es que no son exógenas a las características del sistema de partidos sobre el cual impactarían. Particularmente en diversos países de América Latina los CFP han sido en tiempos recientes modificados en varias ocasiones con el objetivo de abrir el sistema de partidos ante una crisis de representación, o cerrarlo ante una excesiva fragmentación (Scherlis, 2014). Sin embargo, para los casos analizados en este trabajo los CFP se han mantenido esencialmente constantes desde 1983. En el año 2009 se introdujo una reforma estableciendo que en lugar de un 0,4 por ciento de firmas sea necesario reunir ese mismo porcentaje pero de afiliaciones. Este cambio, de todos modos, fue producto de una reforma de la regulación a nivel nacional, que afectó a los 24 distritos por igual, y que no puede ser alterada por los partidos de distrito de una provincia. En otras palabras, los partidos reconocidos en un distrito no tienen capacidad para modificar el requisito de afiliaciones regulado por la ley nacional, por lo que descartamos que exista endogeneidad entre ambas variables. 


\section{Impacto del número de partidos reconocidos sobre la oferta electoral y la fragmentación legislativa}

Comprender las causas de la heterogeneidad en el número de partidos reconocidos en las provincias argentinas es relevante porque esta variable a su vez tiene consecuencias sobre la oferta electoral. Tal como intuitivamente cabe esperar, existe una alta correlación positiva entre la cantidad de partidos inscriptos en un distrito y la cantidad de listas que se presentan a elecciones.

El Gráfico 3 muestra la existencia de una correlación de 0.83 entre el número de partidos reconocidos y de listas presentadas en las elecciones primarias del año $2017^{8}$.

\section{Gráfico 3}

Partidos de distrito reconocidos y listas presentadas, por provincia (2017) ${ }^{9}$

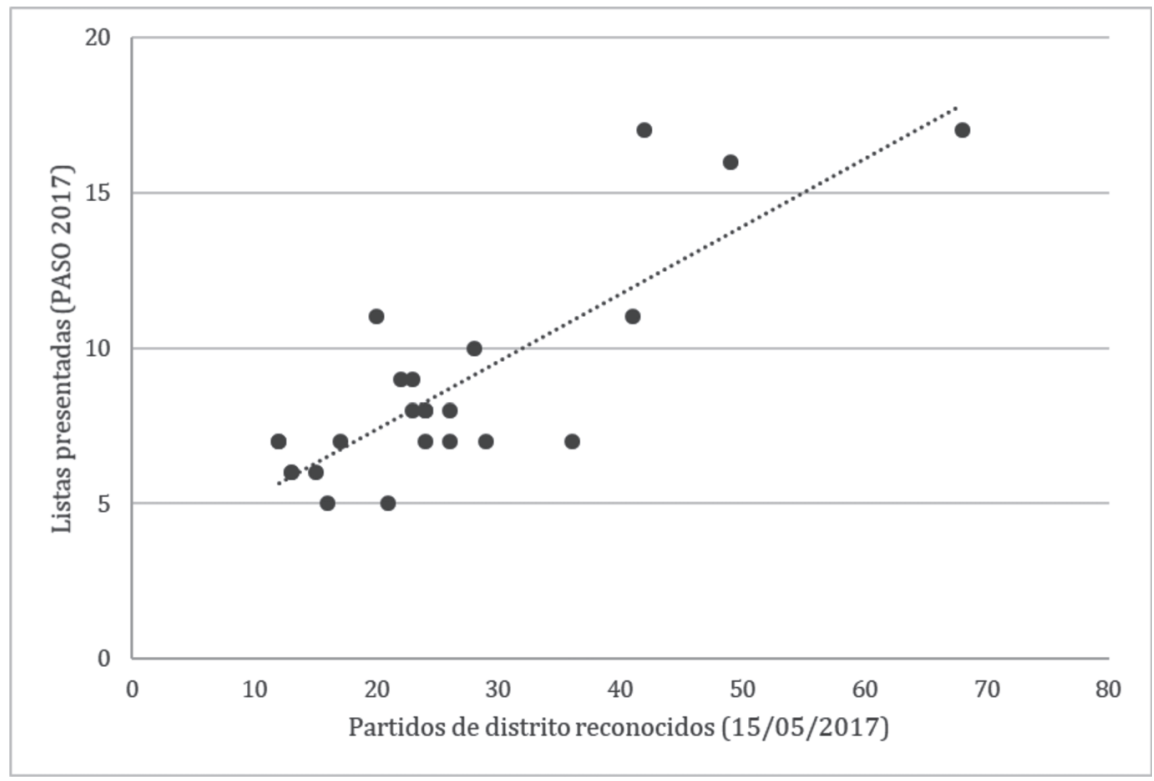

Fuente: elaboración propia en base a datos de la Cámara Nacional Electoral.

Clérici (2016) encuentra esta misma correlación para el período 1983-2013.

Se observan 21 puntos en el gráfico porque se producen varias coincidencias entre distritos: Santa Cruz y La Pampa tuvieron en 201713 partidos reconocidos y 6 listas en competencia, mientras que Salta y Mendoza tuvieron 24 partidos reconocidos y 8 en competencia. 
La línea de tendencia muestra una pendiente positiva, indicando que en las provincias con mayor cantidad de partidos reconocidos encontramos mayor cantidad de listas. El coeficiente de determinación R2 indica que el $70 \%$ de la variación en el número de listas presentadas es explicada estadísticamente por el número de partidos reconocidos.

Otra posible consecuencia del número de partidos reconocidos, mediado por el número de listas presentadas, se da en la conformación de la Cámara de Diputados, para la cual rige una fórmula de distribución proporcional y algunos distritos cuentan con magnitudes grandes y medianas. Esperamos que a mayor cantidad de partidos reconocidos, exista mayor fragmentación en la Cámara de Diputados. En el siguiente gráfico se puede ver la evolución del número de partidos reconocidos en todos los distritos, la cantidad de listas presentadas y el número efectivo de partidos legislativos en la Cámara de Diputados de la Nación.

\section{Gráfico 4}

Partidos de distrito reconocidos, listas presentadas y NEP legislativo Diputados

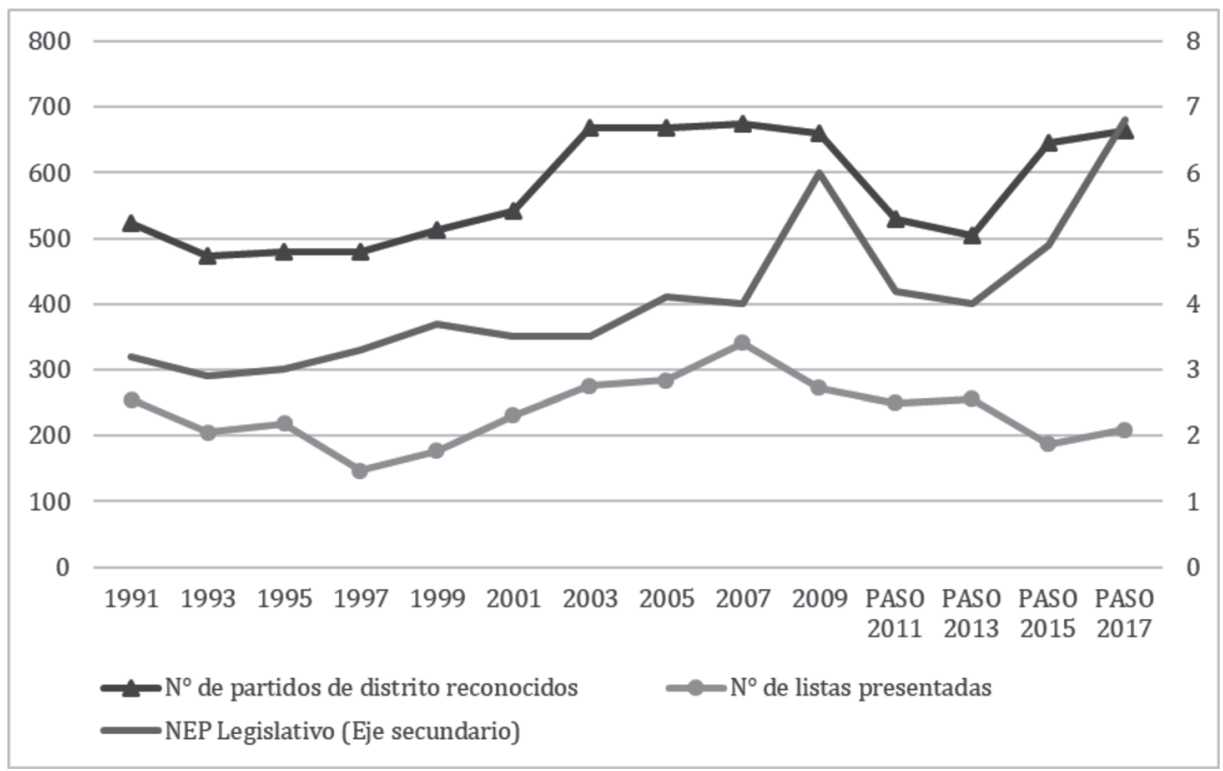

Fuente: elaboración propia en base a datos de la Cámara Nacional Electoral, Clerici (2016) y Mustapic (2013). 
La evolución del número de partidos reconocidos muestra un recorrido muy similar al NEP legislativo en la Cámara de Diputados durante todo el período. El número de listas presentadas, en cambio, toma un recorrido distinto a partir del año 2013. Esto se debe en gran parte al incentivo de agregar partidos en una misma lista que produjeron las PASO (primarias abiertas, simultáneas y obligatorias), ya que establecen un piso mínimo de 1,5 por ciento de los votos válidos para pasar a la elección general.

Sin embargo, como hemos mostrado, a nivel distrito existe una alta correlación entre partidos reconocidos y listas presentadas.

\section{Conclusiones}

Suele decirse que Argentina cuenta con muchos partidos reconocidos. A menudo se responsabiliza por esta situación a una legislación que en lo que respecta a los CFP sería excesivamente permisiva. Sin embargo, no se ha advertido que la distribución territorial de los partidos reconocidos es muy desigual entre los distritos del país, en un rango que va desde 12 hasta 75 partidos por distrito. De igual modo, tampoco se ha considerado el hecho de que los CFP son variables dependiendo del número de electores del distrito.

En esta primera aproximación, precisamente se halló que el CFP es la variable que mejor explica estadísticamente la variación en el número de partidos por distrito. Más específicamente, el descenso en el porcentaje de afiliados sobre el padrón necesario para constituir un partido explica el aumento en el número de partidos reconocidos.

Identificar esta disparidad en el número de partidos por distrito y comprender sus causas resulta relevante para comprender una característica central y relevante del sistema político argentino. Esta característica adquiere particular importancia al advertir que el número de partidos registrados impacta sobre el número de fuerzas políticas que compiten en elecciones en cada distrito e, indirectamente, también sobre la cantidad de bloques legislativos en la Cámara de Diputados.

En consecuencia, creemos que estos hallazgos pueden constituir una importante contribución a los recurrentes debates sobre la fragmentación de la oferta electoral y la fragmentación legislativa en Argentina, así como sobre las posibles reformas legales para eventualmente dar respuesta a esta cuestión. 


\section{Danilo Degiustti y Gerardo Scherlis}

\section{Referencias bibliográficas}

Abal Medina, J. M. (2010). Manual de Ciencia Política. Buenos Aires, Argentina: Eudeba. Amorim Neto, O., \& Cox, G. (1997). Electoral institutions, cleavage structures, and the number of parties. American Journal of Political Science, 41, 149-174.

Birnir, J. (2004). Stabilizing party systems and excluding segments of society? The effects of formation costs on representation in Latin America. Studies in Comparative International Development, 39(3), 3-27.

Calvo, E., Szwarcberg, M., Micozzi, J. P., \& Labanca, J. F. (2001). Las fuentes institucionales del gobierno dividido en la Argentina: sesgo mayoritario, sesgo partidario y competencia electoral en las legislaturas provinciales argentinas. En J. M. Abal Medina \& E. Calvo. El federalismo electoral argentino: sobrerrepresentación, reforma política y gobierno dividido en la Argentina (pp. 53-98). Buenos Aires: Instituto Nacional de la Administración Pública.

Calvo, E. y M. Escolar (2005). La nueva política de partidos en la Argentina. Crisis política, realineamientos partidarios y reforma electoral. Buenos Aires: Prometeo.

Clerici, P. (2016). La creciente importancia de las alianzas electorales, Revista SAAP, 9(2), 313-341.

Dahl, R. (1991). Los dilemas del pluralismo democrático. DF, México: Alianza Editorial.

Escolar, M. (2014). Juntos pero no revueltos. Segmentación estratégica y coaliciones efímeras, en Escolar, M. y J. M. Abal Medina (Coord.), Modus Vivendi. Política multinivel y estado federal en Argentina (pp. 217-254). Buenos Aires: Prometeo.

Gervasoni, C. (2018a). Hybrid Regimes Within Democracies. Fiscal Federalism and Subnational Rentier States. Cambridge: Cambridge University Press.

Gervasoni, C. (2018b). Argentina's Declining Party System: Fragmentation, Denationalization, Factionalization, Personalization and Increasing Fluidity. En Mainwaring, S., Party Systems in Latin America. Institutionalization, Decay, and Collapse (pp. 255-290), Cambridge: Cambridge University Press.(pp. 255-290).

Gervasoni, C. (2009). Democracia y autoritarismo en las provincias argentinas. Revista Aportes (para el Estado y la Administración Gubernamental), 15(27), 101-116.

Malamud, A. y M. De Luca (2016). ¿Todo sigue igual que ayer? Continuidad y ruptura en el sistema de partidos argentino (1983-2015). En Freidenberg (Ed.), Los sistemas de partidos en América Latina 1978-2015 (pp. 29-68), Tomo 2. México: UNAM.

Giraudy, A. (2010). The Politics of Subnational Undemocratic Regime Production in Argentina and Mexico. Journal of Politics in Latin America, 2(2), 53-84.

Hug, S. (2001). Altering Party Systems: Strategic Behavior and the Emergence of New Political Parties in Western Democracies. Ann Arbor, MI: Michigan University Press.

Jones, M. (1995). Electoral Laws and the Survivial of Presidential Democracies. Notre Dame, IN: Notre Dame University Press.

Jones, M. (1997). Federalism and the Number of Parties in Argentine Congressional Elections. The Journal of Politics, 59(2), 538-549. 
Leiras, M. (2010). Los procesos de descentralización y la nacionalización de los sistemas de partidos en América Latina, Política y Gobierno, XVII (2), 205-241.

Leiras, M. (2007). Todos los caballos del rey. Buenos Aires: Prometeo.

Mustapic, A. M. (2013). Los partidos políticos en la Argentina: Condiciones y oportunidades de su fragmentación. En C. Acuña (Ed.) ¿Cuánto Importan las Instituciones? Gobierno, Estado y Actores en la Política Argentina. Buenos Aires: Siglo XXI.

Mustapic, A. M., Scherlis, G., Page, M. (2011). De colectoras, espejos y otras sutilezas. Claves para avanzar hacia una oferta electoral más transparente. Documento de Políticas Públicas/Recomendación Nº0, CIPPEC, Buenos Aires.

Navarro, M., M. Tieghi y C. Varetto (2013). La imagen de una fragmentación partidaria con desequilibrio regional en la política argentina. Una revisión analítica, Revista Ciencia Política (Colombia), 16, 34-65.

Pomares, J.; Scherlis, G. y Page, M. (2012). La reforma después de la reforma: hacia un sistema de partidos más ordenado y una oferta electoral más transparente. Informe de monitoreo y evaluación, CIPPEC, Buenos Aires, Argentina.

Rosemberg, J. (2018, 8 de marzo). Partidos hasta en la sopa. Diario La Nación. [En línea]. Disponible en: https://www.lanacion.com.ar/2115079-partidos-hasta-enla-sopa.

Sabatini, C. (2003). Latin America's lost illusions: Decentralization and political parties. Journal of Democracy, 14(2), 138-150.

Scherlis, G. (2016). La regulación de los partidos latinoamericanos y su integración territorial. Revista Derecho Electoral, 21, 99-126.

Scherlis, G. (2014). Political Legitimacy, Fragmentation and the Rise of Party-Formation Costs in Contemporary Latin America. International Political Science ReviewIPSR, 35(3), 306-323.

Suárez Cao, J. (2011). ¿Federal en teoría pero unitaria en la práctica? Una discusión sobre el federalismo y la provincialización de la política en Argentina. Revisa SAAP, 5(2), 305-321.

Su, Y.-P. (2015). Party registration rules and party systems in Latin America. Party Politics, 21(2), 295-308.

Taagepera, R. (1999). The Number of Parties as a Function of Heterogeneity and Electoral System. Comparative Political Studies, 32(5), 531-548.

Tavits, M. (2006). Party system change: Testing a model of new party entry. Party Politics, 12(1), 99-119.

Van Biezen, I., \& Rashkova, E. R. (2014). Deterring new party entry? The impact of state regulation on the permeability of party systems. Party Politics, 20(6), 890-903.

Varetto, Carlos (2017). Las múltiples vidas del sistema de partidos en Argentina. Villa María: Eduvim.

Zovatto, D. (2006). Regulación jurídica de los partidos políticos en América Latina. Lectura regional comparada. En D. Zovatto (ed.), Regulación jurídica de los partidos políticos en América Latina, International IDEA, DF, Mexico. 


\title{
Palabras clave
}

partidos políticos - provincias - afiliados - partidos de distrito-fragmentación

\section{Keywords}

Political parties - provinces - affiliates - district parties - fragmentation

\begin{abstract}
Argentina has a comparatively high number of registered parties, the highest number in Latin America. This is usually stressed as a problem for the political system, both in terms of representativeness and of governance. However, little attention has been paid to the territorial heterogeneity of this phenomenon. There have also been no serious attempts to identify its causes. This article aims to contribute to fill this gap.

The article starts by identifying a wide inter-district heterogeneity regarding the number of registered parties. Its main finding is that the different party registration legal requirements established by different provinces is the variable that best addresses this heterogeneity. The research also shows that this variation in the number of parties has an impact on the number of party tickets that run elections for legislative offices.
\end{abstract}

